\title{
Acute inflammation of the stomach mimicking linitis plastica
}

\author{
Tuulia Simpanen ${ }^{1}$, Mark Watson ${ }^{2}$ \\ ${ }^{1}$ Department of Radiology, Royal London Hospital, London, UK; ${ }^{2}$ Department of Surgery, Darent Valley Hospital, Dartford, UK \\ Correspondence to: Tuulia Simpanen, MBBS. Department of Radiology, Royal London Hospital, Whitechapel Road, Whitechapel, London E1 1BB, \\ UK. Email: tuulia.simpanen@doctors.org.uk.
}

Submitted Sep 06, 2018. Accepted for publication Nov 20, 2018.

doi: 10.21037/qims.2018.11.11

View this article at: http://dx.doi.org/10.21037/qims.2018.11.11

We present a rare case of resolved computed tomography (CT) appearance of linitis plastica. A 68-year-old gentleman was referred for an outpatient gastroscopy with a 10 -year history of epigastric pain and reflux symptoms. Gastroscopy showed moderate gastritis throughout the stomach. A rapid urease test was negative, and no biopsies were taken. Several hours after the gastroscopy, the patient began experiencing severe abdominal pain at home and 2 days later he presented to the Emergency Department. An abdominal CT scan with iodine contrast was performed to rule out perforation and this showed free fluid in his abdomen and pelvis, but no free air. However, the entire stomach wall appeared grossly thickened, including the antrum and the first part of the duodenum (Figure 1A,B). There was no locoregional nodal disease, retroperitoneal lymphadenopathy or metastatic disease in the abdomen or pelvis. The patient was acidotic and extremely unwell, so he was taken to theatre for an exploratory laparotomy. In theatre, cloudy free fluid and an enlarged grossly thickened stomach were seen. The anaesthetist attempted insufflating the stomach with air but found it very stiff; the thickened appearance to the stomach wall was consistent with linitis plastica, as suggested by the CT scan.

The patient was septic and blood cultures subsequently demonstrated that he had Group A Streptococcal septicaemia, which explained his deterioration. The source of sepsis was thought to be likely Streptococcal pharyngitis, as it turned out that the patient had an upper respiratory tract infection 1 week prior to the gastroscopy. Postoperatively, the patient was initially treated on the intensive care unit with clindamycin and subsequently Tazocin; he required inotrope and renal support, but made a full recovery within the following few weeks. Gastroscopy was repeated a month later, and this showed a grossly ulcerated stomach. Biopsies taken in the second gastroscopy showed only inflammatory changes and sloughing. As per tertiary centre advice in view of curative surgery, a CT scan of the thorax was performed for staging purposes. This CT scan of the thorax included the fundus and the body of the stomach, revealing significant regression of the previously noted gastric wall thickening (Figure 1C,D). The patient was discussed in the upper gastrointestinal malignancy multidisciplinary team meeting, and it was concluded that he had no malignancy. This was confirmed by a follow-up CT abdomen with contrast 3 months later (Figure 1E,F), by which the patient was completely asymptomatic and back to full functioning. The changes seen on the first CT scan, which were in keeping with linitis plastica, seemed to have been caused by an inflammatory process. Without having known the previously made diagnosis of linitis plastica, the appearances of the stomach on the staging CT thorax would have been considered normal.

Linitis plastica, or 'leather bottle stomach' as it is sometimes described, is a gastric cancer of diffuse histotype characterised by thickening of the stomach wall and deformation of the stomach (1). The most common cause of linitis plastica is primary gastric carcinoma. Metastatic gastric cancer secondary to extensive serosal disease from omental metastases and many other metastatic malignancies, such as non-Hodgkin lymphoma involving the stomach, have also been reported to demonstrate radiographic findings similar to those of linitis plastica $(2,3)$. However, in a review of literature, we found no reports of gastric linitis plastica secondary to inflammation, although appearances 

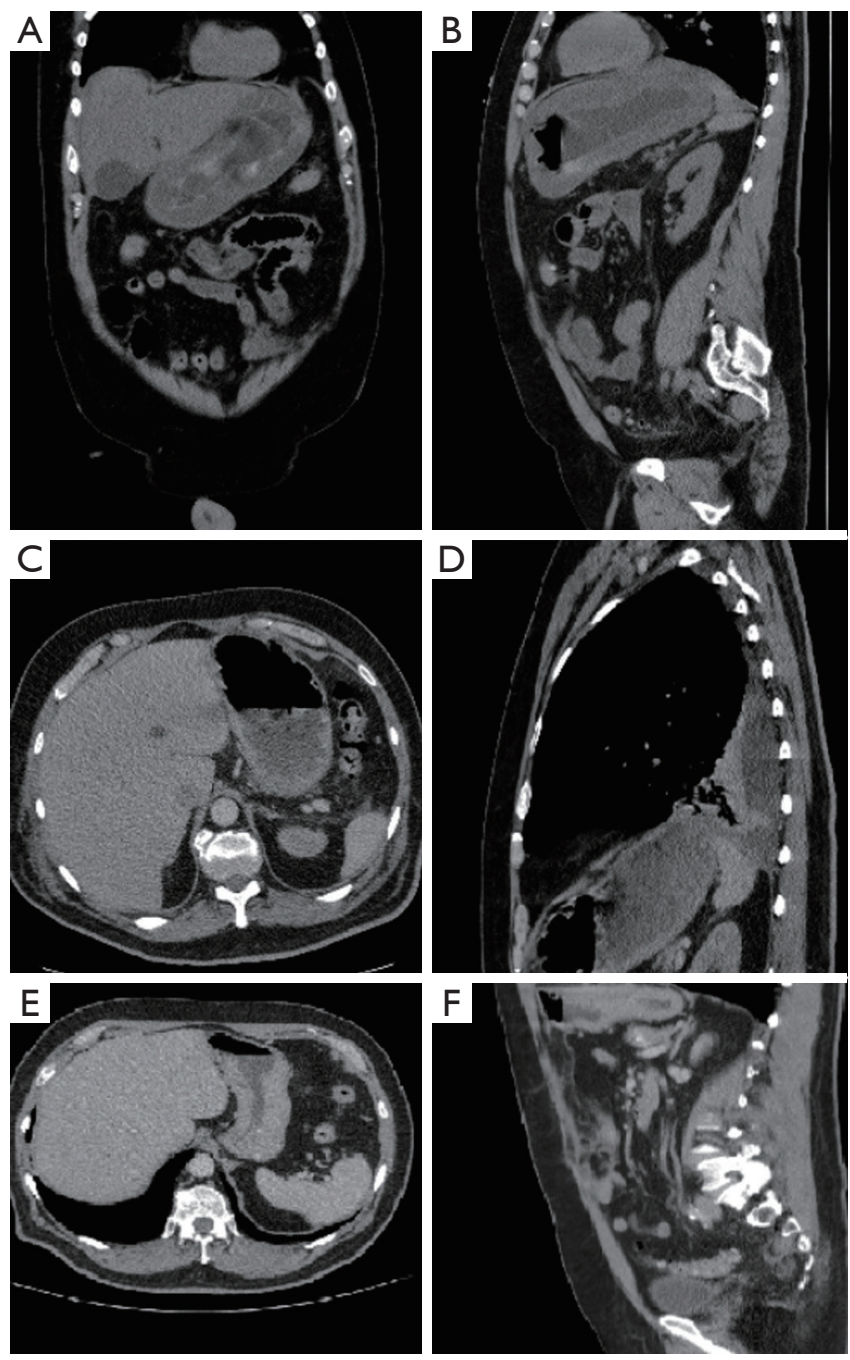

Figure 1 Coronal reformat (A) of the CT scan of abdomen and pelvis performed on admission, shows the grossly thickened gastric wall. These findings are also demonstrated in a sagittal view (B). The contrast-enhanced CT scan (C,D) of the thorax, performed 3 weeks after the first CT scan, displays significant regression of the previously noted gastric wall thickening. Follow-up CT scan of abdomen with contrast (E,F), performed 3 months after the first scan, confirms resolved appearances of the stomach wall.

Cite this article as: Simpanen T, Watson M. Acute inflammation of the stomach mimicking linitis plastica. Quant Imaging Med Surg 2018;8(11):1121-1122. doi: 10.21037/ qims.2018.11.11 of linitis plastica secondary to inflammation have been reported in the rectum (4). In conclusion, radiological appearances synonymous to advanced gastric cancer may be seen secondary to inflammation or infection in the stomach, and hence should be included in the differential diagnosis of linitis plastica.

\section{Acknowledgements}

None.

\section{Footnote}

Conflicts of Interest: The authors have no conflicts of interest to declare.

Informed Consent: Written informed consent was obtained from the patient for the publication of this manuscript and any accompanying images.

\section{References}

1. Maeda E, Oryu M, Tani J, Miyoshi H, Morishita A, Yoneyama H, Kobara H, Mori H, Masaki T. Characteristic waffle-like appearance of gastric linitis plastica: A case report. Oncol Lett 2015;9:262-4.

2. Hong WS, Chung DJ, Lee JM, Byun JH, Hahn ST. Metastatic gastric linitis plastica from bladder cancer mimicking a primary gastric carcinoma: a case report. Korean J Radiol 2009;10:645-8.

3. Tseveldorj N, Koklu S, Katipoglu K. An Unusual Cause of Gastric Linitis Plastica. Gastroenterology 2016;151:e10-1.

4. Keogh CF, Brown JA, Phang PT. Linitis plastica of the rectum: utility of transrectal ultrasonography. J Ultrasound Med 2002;21:103-6. 